\title{
Percepção de pacientes em tratamento oncológico ambulatorial sobre o ato de se alimentar
}

\author{
Perception of oncological outpatients about the act of feeding \\ Percepción de pacientes en tratamiento oncológico ambulatorio en el acto de alimentación \\ Sabina Gonçalves Rodrigues ${ }^{1}$, Flávia Stephanie Baía Teixeira ${ }^{1}$, Gisele dos Santos Martins ${ }^{1}$, Lorena \\ Furtado Falcão1, Thaís de Oliveira Carvalho Granado Santos ${ }^{2 *}$, Ana Claudia Ferreira do Valle ${ }^{2}$, \\ Amanda Larissa Garça de Souza ${ }^{3}$.
}

\section{RESUMO}

Objetivo: Avaliar a percepção de pacientes em tratamento oncológico ambulatorial sobre 0 ato de se alimentar, investigar as mudanças alimentares e como enfrentam as restrições físicas e alimentares dos pacientes em relação a doenças e/ou tratamento. Métodos: Trata-se de estudo transversal, descritivo e quantiqualitativo, realizado com pacientes adultos, em acompanhamento nutricional em um hospital oncológico em Belém-Pará. Foi aplicado formulário com oito perguntas sobre a percepção do ato de se alimentar frente aos desafios impostos pelo tratamento oncológico, dados clínicos e sociodemográficos. Resultados: Participaram 24 pacientes oncológicos entre 29 e 80 anos, sendo a maioria adulto (70,8\%). Houve prevalência de mulheres $(79,2 \%)$, com câncer de mama $(45,8 \%)$ e em tratamento pós-cirúrgico $(58,3 \%)$. Alterações fisiológicas e/ou sensoriais afetam a alimentação do paciente, causando insatisfação e tristeza com a mudança alimentar. A saúde comprometida, os agravos ocasionados pelo tratamento e as mudanças na alimentação têm impacto emocional, levando a maioria a buscar apoio da religião, família, amigos ou profissionais de saúde (95,2\%). Conclusão: As mudanças na alimentação e as restrições impostas ocasionam insatisfação e têm influência na qualidade de vida, embora a maioria concorde que a alimentação adequada diminui os agravos e está relacionada à recuperação.

Palavras-Chave: Ingestão de alimentos, Neoplasias, Percepção.

\section{ABSTRACT}

Objective: To evaluate the perception of outpatients undergoing cancer treatment about the act of feeding, to investigate dietary changes regarding and how they deal with patients' physical and dietary restrictions in relation to diseases and / or treatment. Methods: This is a cross-sectional, descriptive, quantitative-qualitative study, conducted with adult patients undergoing nutritional monitoring at an oncology hospital in Belém, Pará. A form with eight questions about the perception of the act of feeding in the face of the challenges imposed by cancer treatment, clinical and sociodemographic data was applied. Results: Twenty-four cancer patients aged 29 to 80 years participated, the majority being adults (70.8\%). There was a prevalence of women (79.2\%), patients with breast cancer (45.8\%) and undergoing post-surgical treatment (58.3\%). Physiological and/or sensory changes affect patient's diet, causing dissatisfaction and sadness with the dietary changes. Compromised health and the health problems caused by treatment and changes in the diet have an emotional impact leading the majority to seek support from religion, family, friends or health professionals (95.2\%). Conclusion: Changes in diet and the restrictions imposed cause dissatisfaction and have an influence on life quality, although most agree that an adequate nutrition reduces the problems and is related to recovery.

Keywords: Eating, Neoplasms, Perception.

1 Universidade da Amazônia (UNAMA), Belém - PA. *E-mail: thaissoliveira@yahoo.com.br

2 Hospital Ophir Loyola (HOL), Belém - PA.

3 Universidade do Estado do Pará (UEPA), Belém - PA. 


\section{RESUMEN}

Objetivo: Evaluar la percepción de los pacientes sometidos a un tratamiento oncológico y ambulatorio acerca de la alimentación, investigar los cambios en la dieta y cómo hacen frente a las restricciones físicas y dietéticas de los pacientes en relación con la enfermedad y/o el tratamiento. Métodos: Este es un estudio transversal, descriptivo y cuantitativo-cualitativo, realizado con pacientes adultos y en monitoreo nutricional en la clínica ambulatoria de nutrición del Hospital de oncología Ophir Loyola en Belém-Pará. Se aplicó un formulario con ocho preguntas sobre la percepción del acto de comer frente a los desafíos impuestos por el tratamiento del cáncer, los datos clínicos y sociodemográficos. Resultados: Veinticuatro pacientes oncológicos de 29 a 80 años participaron en el estudio, $(70.8 \%)$ adultos y $(29.2 \%)$ ancianos. La mayoría eran mujeres $(79,2 \%)$, la prevalencia de cáncer de mama $(45.8 \%)$ y en tratamiento post quirúrgico $(58,3 \%)$. Se observó entre los participantes y también se encontraron tumores ginecológicos $(20.8 \%)$, del tracto gastrointestinal $(20.8 \%)$, de cabeza y cuello $(8.3 \%$ ) y urológico $(4.2 \%)$. Los cambios fisiológicos y / o las sensaciones afectan la dieta del paciente, causando insatisfacción y tristeza con el cambio en la dieta. La salud comprometida, las quejas causadas por el tratamiento y los cambios en la dieta tienen un impacto emocional, y la mayoría busca ayuda de la religión, la familia, los amigos o los profesionales de la salud (95.2\%). Conclusión: Los cambios en la dieta y las restricciones impuestas causan insatisfacción e influyen en la calidad de vida, aunque la mayoría está de acuerdo en que una nutrición adecuada reduce las lesiones causadas por la enfermedad y está relacionada con la recuperación.

Palabras clave: Ingestión de alimentos, Neoplasias, Percepción.

\section{INTRODUÇÃO}

O câncer é uma enfermidade multicausal caracterizada pelo crescimento desordenado, rápido e invasivo de células, com alta capacidade de divisão e de expansão para outros tecidos, podendo ocasionar efeitos agressivos no organismo e transtornos funcionais.

As causas para o desenvolvimento da carcinogênese estão relacionadas a fatores externos, internos ou podem estar inter-relacionadas (COZZOLINO SMF e COMINETTI C, 2013). Um terço da mortalidade por câncer está associada a riscos comportamentais e alimentares, como alto índice de massa corporal, baixo consumo de frutas e vegetais, falta de atividade física e uso de álcool e tabaco (OPAS, 2018).

Dentre as causas observadas nos estudos a respeito da incidência da doença estão, o contato com substâncias químicas, o tabagismo, a poluição ambiental, a desigualdade econômica e o crescente processo de industrialização (LOPES ER, et al., 1992), o qual afetou, sobremaneira, a condição nutricional dos indivíduos, consequência dos novos hábitos praticados e comportamentos alimentares adquiridos (OPAS, 2018).

A alimentação é considerada um dos fatores determinantes para o desenvolvimento de doenças. As práticas alimentares adquiridas na infância e na adolescência podem atuar diretamente sobre o risco de desenvolvimento de câncer, que pode ocorrer quando há exposição cumulativa a substâncias carcinogênicas, também como a insuficiência de substâncias protetoras na alimentação. Desta maneira, a alimentação pode implicar em efeitos que alterem as respostas metabólicas e hormonais que estão relacionadas ao balanço energético corporal (JONES LA, et al., 2006).

A relação entre o câncer e os fatores alimentares é bastante complexa e depende de características como os tipos de alimentos consumidos, componentes específicos de cada um deles, os métodos de preparo, a quantidade das porções, conservação, entre outras. Vale lembrar, que, o sobrepeso e a obesidade são apontados como a segunda causa evitável de câncer, o que exalta a importância da prática de hábitos alimentares mais saudáveis durante toda a vida (COLDITZ GA, et al., 2006).

Por outro lado, a globalização e o acesso a novas tecnologias possibilitam meios para um melhor diagnóstico e tratamento, assim como, um maior mapeamento e registro dos números epidemiológicos, incentivando investimentos no diagnóstico e nas terapias (BITENCOURT ER, et al., 2013). 
O câncer é responsável por cerca de $12 \%$ de todas as causas de óbito no mundo, correspondendo a mais de 7 milhões mortes anuais. Se em 2002, eram estimados 11 milhões de casos novos, em 2020 a estimativa é que a incidência alcançará mais de 15 milhões (INCA, 2019).

A doença influencia diretamente no estado nutricional do indivíduo, e o quanto isso irá impactar na sua saúde depende de inúmeros fatores. A desnutrição em indivíduos com câncer é muito frequente, sendo ocasionada principalmente por conta dos efeitos colaterais relacionados a doença e ao tratamento oncológico, como a redução do apetite, dificuldades mecânicas para mastigar e deglutir alimentos, redução na ingestão de alimentos e alterações metabólicas (MOREIRA JC e WAITZBERG DL, 2000).

O indivíduo diagnosticado e em tratamento oncológico enfrenta vários impactos e mudanças no seu cotidiano, nos âmbitos emocional, familiar e físico. Para muitos pacientes o câncer está relacionado a incapacidade, a um sofrimento lento, doloroso e até mesmo a morte. A atuação de uma equipe multidisciplinar e o apoio familiar é fundamental para o cuidado e aceitação do paciente, pois minimiza seus medos e anseios perante as mudanças ocorridas nesta fase (INCA, 2017).

Frente a tantos desafios impostos pelo tratamento, a dedicação do paciente é fundamental, por meio da força de vontade, comprometimento e busca por estratégias minimizem os efeitos colaterais. $E$ isto pode se tornar ainda mais benéfico quando aliado a bons hábitos alimentares que contribuirão para o bem-estar e 0 resgate da saúde do paciente (BAIOCCHI O, et al., 2018).

$O$ ato de se alimentar cria uma ligação, uma relação com o alimento e a forma com a qual o indivíduo escolhe o alimento mostra quem ele é, de onde veio e como está se sentindo. Quando se come um alimento, agrega-se um valor emocional a ele onde se resgata memórias, lembranças e consequentemente sensações. (WILSON B, 2014).

Nesse sentido, o objetivo deste estudo foi analisar a percepção de pacientes oncológicos sobre 0 ato de se alimentar frente aos desafios impostos pelo tratamento em um ambulatório de hospital oncológico investigando as mudanças alimentares, reconhecendo os mitos e tabus, verificando as restrições alimentares impostas pelo tratamento e como as enfrentam.

\section{MÉTODOS}

Caracteriza-se como um estudo transversal, descritivo com abordagem qualitativa e quantitativa, realizado com pacientes oncológicos, adultos, de ambos os sexos, que realizavam acompanhamento nutricional no ambulatório de um hospital de referência em oncologia em Belém-PA, cuja coleta de dados ocorreu em dezembro de 2019. Para participar do estudo, os pacientes precisavam ter boa capacidade de compreensão e de comunicação e autorizar a participação na pesquisa por meio do Termo de Consentimento Livre Esclarecido (TCLE). A pesquisa foi aprovada pelo Comitê de Ética em Pesquisa do Hospital Ophir Loyola sob número CAAE 22883019.6.0000.5550 e seguiu as normas de pesquisa com seres humanos conforme estabelece a Resolução CNS 466/2012.

A coleta de dados foi realizada por meio de formulário semiestruturado contendo dados clínicos, sociodemográficos e oito perguntas sobre a percepção do ato de se alimentar frente aos desafios impostos pelo tratamento oncológico ambulatorial, das quais cinco referiam-se aos aspectos qualitativos.

As respostas foram organizadas em um banco de dados no Microsoft Office Exce/ versão 16.32. Os dados quantitativos foram analisados por meio da estatística descritiva sendo apresentados sob a forma de tabelas. O conteúdo dos discursos referentes aos aspectos qualitativos foram analisados por meio Plataforma IRAMUTEQ versão 0.7 alpha 2, utilizando-se o método Classificação Hierárquica Descendente (CHD).

\section{RESULTADOS E DISCUSSÃO}

\section{Análise quantitativa}

Participaram do estudo 24 pacientes oncológicos na faixa etária de 29 a 80 anos e idade média $54 \pm 12$ anos, sendo $70,8 \%$ adultos e $29,2 \%$ idosos. A maioria era composta por mulheres $(79,2 \%)$ e oriunda da capital, Belém-PA (58,3\%) (Tabela 1). Assim como no presente estudo, o estudo de Mota ES, et al. (2019), 
realizado em um hospital oncológico no Pará, encontrou média de idade semelhante e prevalência do gênero feminino. Estudo realizado no Marrocos também apontou prevalência do gênero feminino, porém a média de idade foi um pouco menor (SAMOUTH Y, et al., 2020).

Tabela 1 - Distribuição da porcentagem e do número de participantes do estudo segundo gênero, idade e origem. Belém - PA, 2019.

\begin{tabular}{ccc}
\hline Variáveis & N & $\%$ \\
\hline Gênero & & \\
\hline Feminino & 19 & 79,2 \\
Masculino & 5 & 20,8 \\
\hline Idade & \\
\hline Adulto & 17 & 70,8 \\
Idoso & 7 & 29,2 \\
\hline Origem & & \\
\hline Capital & 14 & 58,3 \\
Interior & 10 & 41,7 \\
\hline
\end{tabular}

Fonte: RODRIGUES SG, et al., 2020.

Estudo recente mostrou que algumas modificações que envolvem a vida reprodutiva vêm acarretando transformações biológicas direta ou indiretamente relacionadas ao desenvolvimento de tumores em mulheres adultas.

Tais modificações estão associadas com o número de gestações, a idade de início da vida reprodutiva, duração do aleitamento materno, prática de abortamento, o número de parceiros sexuais, entre outros, e são decorrentes tanto da inserção da mulher na esfera do trabalho produtivo como da introdução de medicamentos hormonais (LOFRANO AD, et al., 2016).

Destaca-se o número elevado de participantes oriundos de municípios do interior do estado, que compõe a amostra deste estudo de $(41,7 \%)$, justificado pelo fato do Hospital ser referência no atendimento de pacientes oncológicos na região, possibilitando o diagnóstico precoce, tratamentos e possíveis intervenções no quadro. Isto contribui para um melhor prognóstico, sendo uma das razões pelas quais os indivíduos dos municípios do interior são encaminhados para a capital Belém para seu tratamento (INCA, 2019).

No que se refere à localização do tumor, foi observado a prevalência de câncer de mama $(45,8 \%)$ entre os participantes, sendo ainda encontrado tumores ginecológicos $(20,8 \%)$, do trato gastrointestinal $(20,8 \%)$, de cabeça e pescoço (8,3\%) e urológico (4,2\%) (Tabela 2). Nos estudos de Mota ES, et al. (2019) e Samouth Y, et al., (2020), o câncer de mama também foi prevalente.

Para o triênio 2020-2022 foram estimados 66.280 casos de câncer de mama a cada ano no Brasil, com um risco estimado de 61,61 novos casos a cada 100 mil mulheres. O câncer de útero, que ocupou o segundo lugar entre os entrevistados, foi estimado em 16.590 novos casos para cada ano do biênio 2020-2022, com risco previsto de 15,43 casos a cada 100 mil mulheres, ocupando a terceira posição do País (INCA, 2019).

Em relação ao tipo de tratamento, a maioria relatou estar em tratamento pós-cirúrgico $(58,3 \%)$ no período em que os dados foram coletados (Tabela 2). Os pacientes encontravam-se em acompanhamento ambulatorial com a equipe multiprofissional do serviço, tentando transpor os agravos da doença e os efeitos do tratamento, com destaque para a assistência nutricional, que visa minimizar os sinais e sintomas ocasionados pelo tratamento e garantir uma melhor qualidade de vida ao paciente e para o suporte psicológico, citado pelos participantes como uma alternativa de apoio, pois o prognóstico da doença causa impacto emocional (WILSON B, 2014). 
Tabela 2 - Distribuição da porcentagem e do número de pacientes entrevistados segundo a localização do tumor. Belém-PA, 2019.

\begin{tabular}{ccc}
\hline Variáveis & N & $\%$ \\
\hline Localização do Tumor & & \\
\hline Mama & 11 & 45,8 \\
Ginecológico & 5 & 20,8 \\
Trato Gastrointestinal & 5 & 20,8 \\
Cabeça e Pescoço & 2 & 8,3 \\
Urológico & 1 & 4,2 \\
\hline Tipo de Tratamento & \\
\hline Pré-cirúrgico & 10 & 41,6 \\
Pós-cirúrgico & 14 & 58,3 \\
\hline
\end{tabular}

Fonte: RODRIGUES SG, et al., 2020.

Em relação às perguntas do estudo, a maioria respondeu concordar que se alimentar de forma correta pode diminuir os agravos da doença e os efeitos do tratamento $(95,8 \%)$. Os participantes relataram que têm conhecimento dos benefícios da alimentação para o resgate da saúde e prevenção de outros agravos inerentes a doença. Apenas um paciente $(4,2 \%)$ disse não concordar que alimentação irá diminuir os efeitos do tratamento. Foi visualizado que esta pessoa estava emocionalmente instável e desmotivada e não tinha muito conhecimento sobre a importância da alimentação para seu quadro de saúde (Tabela 3).

A avaliação e o acompanhamento nutricional tem sido enfatizados como importantes no combate à desnutrição e possibilitando melhor qualidade de vida aos pacientes em tratamento oncológico (MOTA ES, et al., 2019). No estudo realizado no Marrocos, a maioria dos participantes nunca tinha recebido orientação nutricional. Para Samouth Y, et al., (2020), o monitoramento do estado nutricional é tarefa multidisciplinar.

As terapias instituídas durante o tratamento oncológico, associadas às alterações ocasionadas pela doença, podem causar efeitos adversos que influenciam diretamente o consumo alimentar e podem impactar a qualidade de vida do paciente (BRITO DA e MAYNARD DC, 2019). Por isso, a boa nutrição é ainda mais importante para as pessoas com câncer. Quem se alimenta adequadamente durante o tratamento tem mais capacidade de vencer os efeitos colaterais deste, e enfrentar com êxito a administração de doses mais altas de medicamentos (PRADO BBF, 2014).

Observa-se ainda que todos os participantes declararam que sua recuperação depende do esforço para aderir a nova recomendação alimentar, o que mostra que os participantes têm consciência da importância do alimento para sua recuperação (Tabela 3).

Tabela 3 - Questionário de avaliação referente a adesão às novas recomendações alimentares propostas durante o tratamento oncológico. Belém-PA, 2019.

\begin{tabular}{cccc}
\hline \multicolumn{2}{c}{ Questões } & N & $\%$ \\
\hline $\begin{array}{c}\text { Você concorda que se } \\
\text { alimentar de forma correta vai } \\
\text { diminuir os agravos da doença } \\
\text { e os efeitos do tratamento? }\end{array}$ & Sim & 23 & 95,8 \\
\hline & Não & 1 & 4,2 \\
\hline $\begin{array}{c}\text { Você tem recebido algum } \\
\text { apoio emocional? }\end{array}$ & Sim, psicológico & 3 & 12,5 \\
& Sim, família & 2 & 8,3 \\
& Sim, psicólogo e outro & 5 & 20,8 \\
$\quad$ Sim, família e outro & 4 & 16,7 \\
$\begin{array}{c}\text { Você acredita que sua } \\
\text { recuperação depende do seu }\end{array}$ & $\begin{array}{c}\text { esforço outros } \\
\text { recomendaçália, religião }\end{array}$ & 9 & 37,5 \\
\hline
\end{tabular}

Fonte: RODRIGUES SG, et al., 2020. 
A qualidade de vida do paciente oncológico está diretamente relacionada a percepção do indivíduo com seu meio. Essa percepção abrange dois processos paralelos, que são independentes, mas devem estar em contínua interação: a percepção cognitiva pautada na representação mental dos elementos da enfermidade e desenvolvimento de ações para controle da doença (enfrentamento); e a percepção emocional, que surge a partir da enfermidade e do valor de determinadas estratégias de enfrentamento para lidar com as emoções (QUILES M, et al., 2009).

Quando questionados se têm recebido algum apoio emocional, a grande maioria dos participantes declarou buscar algum apoio emocional citando a família, a religião, o apoio psicológico, separadamente ou em conjunto. Além de todo cuidado, o apoio da equipe multiprofissional é de extrema importância, o acompanhamento psicológico ajudará a amenizar certas situações e recuperar a saúde mental do paciente. O apoio da família e amigos é essencial para fortalecer o paciente a enfrentar seus sentimentos e seguir ao tratamento (SANCHEZ KOL, et al., 2010).

\section{Análise qualitativa}

Para melhor compreender a percepção dos pacientes sobre o ato de se alimentar, suas falas foram analisadas por meio da Classificação Hierárquica Descendente (CHD), método que classifica os segmentos de texto de acordo com os seus vocabulários, cujo conteúdo é categorizado em função da frequência (CAMARGO BV e JUSTO AM, 2013). O conteúdo das falas foi categorizado em 4 classes conforme pode ser visualizado pelo dendograma (Figura 1).

Figura 1 - Dendograma da Classificação Hierárquica Descendente dos conteúdos das falas de pacientes oncológicos sobre o ato de se alimentar. Belém-PA, 2019.

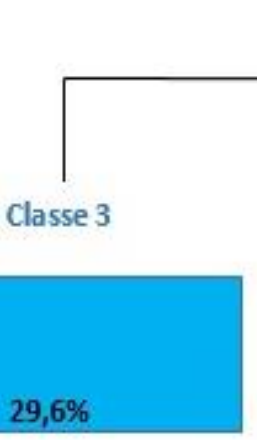

gostar
alimentar
tratamento
conseguir
estar
fruta
feijão
enjoo
contar
causa
prazer
bem

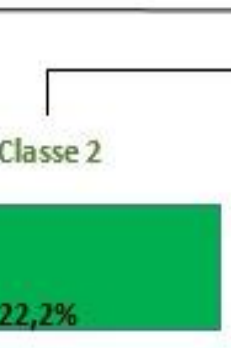

insatisfação
necessidade
alimentação
alimento
fome
mudar
sentir
suprir
corpo
como
tudo
năo
falta

Fonte: RODRIGUES SG, et al., 2020.

\section{Classe 4}
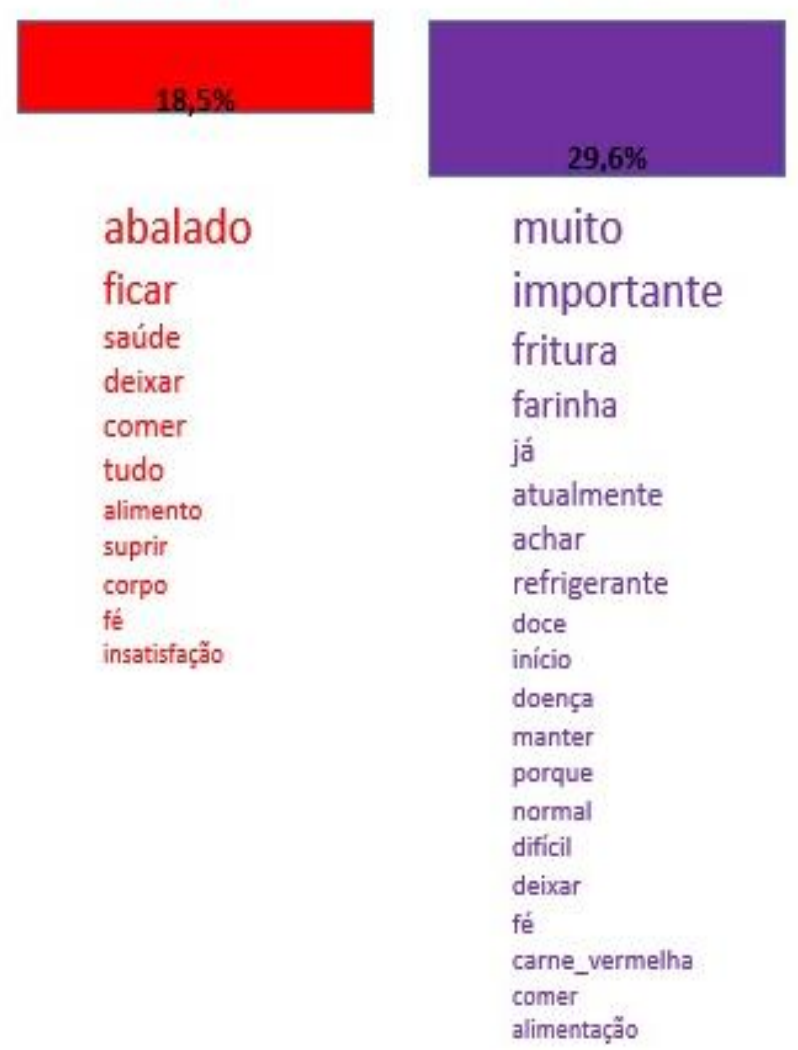


\section{Classe 1 - Comportamento dos pacientes frente aos desafios do câncer.}

A Classe 1 representa um total de 18,5\% segmentos analisados, está ligada aos aspectos emocionais dos pacientes, a palavra de destaque principal é "Abalado". Foi identificado nesta pesquisa que os pacientes se encontram comprometidos com o seu atual quadro de saúde, pois o câncer tem um forte impacto na questão psicológica e emocional (AZEVEDO D, et al., 2017).

Os tratamentos oncológicos como a quimioterapia, radioterapia e mastectomia em casos de câncer de mama, deixam os pacientes "abalados", muitos estão insatisfeitos com sua atual alimentação, com as restrições alimentares que o tratamento impõe, e com o novo estilo de vida. Praticamente todos encontramse em um estado desmotivacional para continuar os tratamentos por uma série de fatores que causa ao organismo e na vida social destes pacientes.

"Estou muito abalada, desde quando descobri que teria que retirar a mama, minha autoestima ficou muito abalada" (Paciente 15).

"Meu psicológico está muito abalado. A minha vida não é mais a mesma desde que iniciei o tratamento" (Paciente 7).

"Só sabe quem passa por essa situação. É muito difícil" (Paciente 3).

"Não sinto vontade de comer nada, a gente perde o apetite e come porque tem que comer". (Paciente 2).

Os relatos dizem respeito a palavra "abalado", que retrata que os pacientes estão com muitas dificuldades para o enfrentamento do tratamento, porém a ajuda psicológica iria amenizar esse sentimento. Segundo Azevedo D, et al. (2017), a figura do psicólogo no contexto hospitalar é tão fundamental quanto às outras formas de acompanhamento, pois o terapeuta ameniza o sofrimento, a angústia e a solidão nesse momento de grave desgaste emocional. Muitas vezes é necessário interpretar através da escuta e do acolhimento, as emoções afloradas do paciente, da família e dos membros da equipe, dando suporte emocional a todos, e por fim, permitir o sucesso do tratamento ou a preparação para a morte como parte da vida.

Diante deste cenário e dos dados analisados no presente estudo, identificou-se que o acompanhamento psicológico é muito importante nesta fase, pois irá contribuir para inserção do paciente à sociedade, possibilitando o resgate emocional, uma vez que, o câncer parece indicar a possibilidade de limite no tempo de vida, e em alguns casos pode resultar em morte (SILVA SS, et al., 2008).

\section{Classe 2 - Percepção dos pacientes quanto as mudanças na alimentação.}

Na classe 2, a palavra "Insatisfação" teve considerável evidência. Outras palavras como "Necessidade" e "Alimentação" também tiveram relevância. Nessa abordagem, o aspecto identificado está correlacionado às mudanças na alimentação que este paciente enfrenta e que ocasiona insatisfação em relação as suas necessidades nutricionais e físicas, ora imposta pela conduta terapêutica. A preocupação, a ansiedade, o medo podem comprometer seu estado e influenciar o consumo alimentar, problemática que está diretamente ligada a classe 1, pois visa o aspecto emocional (GARÔFOLO A, et al., 2004).

"Estou insatisfeita com a minha alimentação mas tenho consciência que é importante para minha melhora" (Paciente 7).

"É uma necessidade comer, mas é complicado quando não se tem vontade, principalmente dentro do hospital" (Paciente 8).

"Eu tinha uma alimentação muito boa e sei que isso me ajuda no tratamento, hoje eu tento manter uma alimentação equilibrada porque eu sei que é importante" (Paciente 3).

Os relatos contemplam o destaque que teve a palavra "insatisfação", uma vez que os participantes se encontravam insatisfeitos com a mudança alimentar, porém conscientes com a "necessidade" desta modificação na dieta. 


\section{Classe 3 - Interferência do tratamento oncológico no hábito alimentar dos pacientes.}

A classe 3 referente a 29,6\% das falas, resultou uma maior frequência nas palavras "Gostar", "Alimentar", "Tratamento" e "Conseguir". Palavras que se ligam com o poder de escolha, pois o tratamento interfere nestas ações, na qualidade de vida do paciente.

Foi observado, no decorrer da pesquisa, que os efeitos causados pelo tratamento oncológico pré e pósoperatório podem ocasionar mudanças fisiológicas e/ou sensitivas no paladar e no olfato, além de náuseas e vômitos. O enfrentamento das mudanças, causa insatisfação, como podemos analisar nas falas a seguir:

"Mudou tudo, não consigo me alimentar por conta dos enjoos que o tratamento causa" (Paciente 9).

"O mal-estar do tratamento tá interferindo na minha alimentação, sinto muito enjoo e vômito" (Paciente 24).

O estudo de Samouth Y, et al., (2020) apontou que a percepção da influência de alguns alimentos na saúde e os efeitos adversos do tratamento foram as principais causas para a mudança no comportamento alimentar. No estudo, os participantes referiram deixar de consumir carne vermelha, produtos lácteos, ovos e peixes. O aparecimento de dores, desconfortos, azia e diarreia, é bastante comum especialmente após as refeições (VALLE TD, et al., 2017), como podemos observar nas falas a seguir:

\footnotetext{
"Não consigo comer, tudo que como causa dor" (Paciente 6).

"Não consigo me alimentar direito" (Paciente 9).

"Tive mudanças na textura da comida, comecei a comer comida pastosa" (Paciente 13).
}

Este resultado retratou uma forte e indissociável ligação dos efeitos causados pelo tratamento oncológico na alimentação, gerando consequências na rotina e na aceitabilidade alimentar do paciente (GARÔFOLO A, et al., 2004).

\section{Classe 4: Visão dos pacientes quanto as restrições alimentares}

A classe 4 apresentou o percentual de $29,6 \%$, as falas em destaque foram, "Muito", "Importante", "Fritura" e "Farinha", palavras que denotam a importância, o valor afetivo e a preferência dada para determinados alimentos. O paciente ao iniciar o tratamento nutricional muitas vezes carrega hábitos alimentares inadequados e possui noções infundadas sobre alimentos específicos, isso se dá pela divulgação dos meios de comunicação e pelos mitos e tabus.

"Eu acredito que a carne de porco é remosa, é um alimento maléfico para saúde" (Paciente 04).

"Acredito que o chá da graviola faz bem para o câncer e os mariscos são alimentos remosos" (Paciente 06).

Acima o relato de pacientes que acreditam que alguns alimentos podem agravar ou melhorar o seu quadro de saúde. Na cultura popular, acredita-se que alguns alimentos possam interferir diretamente nos processos cicatriciais e por isso são contraindicados em situações de ferimentos e no pós-operatório. Estes são conhecidos popularmente como "remosos", como por exemplo a carne de porco e o camarão (MIRANDA TV, et al., 2013).

O chá também foi muito citado entre os entrevistados, especialmente o chá de graviola, que os participantes acreditam ter muitos benefícios contra o câncer. Estudo norte-americano demostrou que um conjunto de ativos fitoquímicos chamados Acetogeninas anonáceas, presentes na folha de graviola, causam toxicidade às células cancerígenas e pouca toxicidade às células saudáveis do organismo, resultando em poucos efeitos colaterais e mais efeito terapêutico. Quando comparada ao fármaco adriamicina, medicamento antitumoral usado para matar células cancerosas e atrasar ou reduzir o crescimento do tumor, a toxicidade às células saudáveis do organismo é bem baixa, o que resulta em poucos efeitos colaterais. Nesse sentido, 
o órgão americano de regulamentação de alimentos e medicamentos, Food and Drug Administration (FDA), aprova somente a utilização da graviola como coadjuvante no tratamento na melhora da função imunológica (LORENZI H e MATOS FJA, 2008).

A preferência e o consumo de farinha foram observados na pesquisa, sendo um alimento presente na maioria das refeições. A mandioca é caracterizada por sua adaptabilidade a diversos climas e regiões, e sua farinha é considerada fonte de carboidratos e está presente na casa na maioria dos brasileiros devido ao seu baixo valor de custo (CHIELLE ZG, et al., 2009).

"Não devo comer farinha, por conta disso afetou bastante meu emocional" (Paciente 1).

"Deixei de comer farinha, refrigerante e fritura" (Paciente 5).

"Cortei a farinha da minha alimentação" (Paciente 3).

Pode-se analisar nas falas que estes pacientes tinham um apego à farinha de mandioca, a qual muitos sentem falta nas refeições do dia a dia. Os pacientes optaram pela sua exclusão da alimentação e alguns enfatizaram seu descontentamento com a atitude, pois o alimento para muitos era considerado muito importante para a sua satisfação emocional e degustativa. Gimenes-Minasse MHSG (2016), descreve essa sensação de conforto e alívio que a comida pode proporcionar, principalmente em momentos de fragilidade.

Embora reconheçam a importância da alimentação e do estilo de vida saudável, tanto na redução dos agravos ocasionados pela doença e dos efeitos deletérios do tratamento, como atuando positivamente na recuperação e qualidade de vida do paciente, os pacientes estão insatisfeitos com as mudanças cotidianas ocorridas na sua alimentação em função da doença e/ou tratamento.

As alterações fisiológicas e/ou sensoriais afetam a alimentação do paciente oncológico, levando-os a deixar de consumir alimentos preferidos, os quais tinham um valor afetivo importante, causando insatisfação e tristeza por se verem compelidos a mudar seu hábito alimentar. A crença popular de que alguns alimentos podem agravar ou melhorar seu quadro também estava presente, fazendo-os incluir ou excluir alimentos na alimentação cotidiana.

\section{CONCLUSÃO}

Os resultados apontaram que embora reconheçam a importância da alimentação saudável na redução dos agravos e efeitos deletérios ocasionados pela doença e/tratamento, assim como na recuperação e qualidade de vida, os participantes estão insatisfeitos com as mudanças ocorridas na alimentação. Alterações fisiológicas e/ou sensoriais afetam a alimentação do paciente oncológico, levando-os a deixar de consumir alimentos de valor afetivo importante, causando insatisfação e tristeza. A crença popular de que alguns alimentos podem agravar ou melhorar seu quadro também os fazem excluir ou incluir alimentos cotidianamente. Deste modo, o apoio da família, dos amigos, dos profissionais e da religião foi considerado importante, proporcionando maior confiança para a superação dos desafios impostos pelo tratamento.

\section{AGRADECIMENTOS}

Ao Hospital Ophir Loyola e a equipe da Divisão de Nutrição e Dietética por autorizar a realização da pesquisa. Aos pacientes que aceitaram participar do estudo e contribuíram para sua realização.

\section{REFERÊNCIAS}

1. AZEVEDO D, et al. Importância do psicólogo na intervenção da psico-oncologia em mulheres acometidas pelo câncer de mama: I Simpósio científico de práticas em psicologia. Psicologia e Saúde em Debate, 2017; 2(Supl. 1): 12 -15.

2. BAIOCCHI O, et al. Aspectos nutricionais em oncologia. Rio de Janeiro: Atheneu, 2018; 652p.

3. BITENCOURT R, et al. Perfil epidemiológico do câncer na rede pública em Porto Alegre, RS. Revista Brasileira de Cancerologia, 2013; 2(50): 95-101.

4. BRITO DA, MAYNARD DC. Avaliação da relação entre nutrição e câncer: Uma visão do impacto no estado nutricional e qualidade de vida de pacientes oncológicos. Nutrición Clínica y Dietética Hospitalaria, 2019; 39(1): 169-175. 
5. CAMARGO BV, JUSTO AM. IRAMUTEQ: Um software gratuito para análise de dados textuais. Temas em Psicologia, 2013; $21(2):$ 513-518.

6. CHIELLE ZG, et al. Desempenho agronômico de cultivares e seleções de mandioca em Rio Pardo, RS. Pesquisa Agropecuária Gaúcha, 2009; 15(1): 53-56.

7. COLDITZ GA, et al. Epidemiology - identifying the causes and preventability of cancer? Nature Reviews-Cancer, 2006; 6(1): 75-83.

8. COZZOLINO SMF, COMINETTI, C. Bases bioquímicas e fisiológicas da nutrição: nas diferentes fases da vida, na saúde e na doença. Barueri: Manole, 2013; 1257p.

9. GARÔFOLO A, et al. Dieta e câncer: um enfoque epidemiológico. Revista de Nutrição, 2004; 17(4): 491-505.

10. GIMENES-MINASSE MHSG. Comfort food: sobre conceitos e principais características. Contextos da Alimentação: Comportamento, Cultura e Sociedade, 2016; 4(2): 92-102.

11. INCA. Instituto Nacional de Câncer José Alencar Gomes da Silva. Laços de família: Apoio ao paciente oncológico é fundamental, mas parentes e amigos também precisam de atenção. Revista Rede Câncer; 2017; 39: 12-15.

12. INCA. Instituto Nacional de Câncer José Alencar Gomes da Silva. Estimativa 2020: incidência de câncer no Brasil. Rio de Janeiro: INCA, 2019; 120p.

13. JONES LA, et al. Between and within: international perspectives on câncer and health disparities. Journal of Clinical Oncology, 2006; 24: 2204-2208.

14. LOFRANO AD, et al. Avaliação da qualidade de vida de mulheres com carcinoma do colo do útero em quimioterapia paliativa. Revista Brasileira de Cancerologia, 2016: 62(3): 203-213.

15. LORENZI H, MATOS FJA. Annona muricata L. Plantas Medicinais no Brasil Nativas e Exóticas. 2 ed. Nova Odessa, SP: Instituto Plantarum de Estudos da Flora LTDA, 2008. 67p.

16. LOPES ER, et al. Câncer e meio ambiente. Revista Brasileira de Cancerologia, 1992; 38(1): 35-64.

17. MIRANDA TV, et al. Estado nutricional e qualidade de vida de pacientes em tratamento quimioterápico. Revista Brasileira de Cancerologia, 2013; 59(1): 57-64.

18. MOREIRA JC, WAITZBERG, DL. Nutrição oral, enteral e parenteral na prática clínica. São Paulo: Atheneu, 2000; $p$ $399-410$.

19. MOTA ES, et al. Avaliação do Risco Nutricional de Pacientes Oncológicos Atendidos no Ambulatório da Unacon em um Hospital de Referência por meio da ASG-PPP. Revista Brasileira de Cancerologia, 2020; 65(4): e-15267.

20. OPAS. Folha Informativa - $2018 . \quad$ Câncer, Disponível em: https://www.paho.org/bra/index.php?option=com_content\&view=article\&id=5588:folha-informativacancer\&Itemid=1094. Acesso em: 08 jul. 2020.

21. PRADO BBF. Influência dos hábitos de vida no desenvolvimento do câncer. Ciência e Cultura, 2014; 66(1): 21 -24.

22. QUILES M, et al. Representación de la Enfermedad, Afrontamiento y Ajuste en los Trastornos Alimentarios. Clinica y Salud, 2009; 20(2): 159-175.

23. SAMOUTH Y, et al. Eating behavior among cancer patients: a cross-sectional study. Nutrición Clínica y Dietética Hospitalaria, 2020; 40(1): 82-88.

24. SANCHEZ KOL, et al. Apoio social à família do paciente com câncer: identificando caminhos e direções. Revista Brasileira de Enfermagem, 2010; 63(2): 290-299.

25. SILVA SS, et al. O paciente com câncer: cognições e emoções a partir do diagnóstico. Revista Brasileira de Terapias Cognitivas, 2008; 4(2): 73-89.

26. VALLE TD, et al. Fatores intervenientes para o início do tratamento de pacientes com câncer de estomago e colorretal. Revista Latino-Americana de Enfermagem, 2017; 25: e2879.

27. WILSON, B. Como Aprendemos a Comer: Por que a alimentação dá tão errado para tanta gente e como fazer escolhas melhores. Rio de Janeiro: Zahar. 2014; 351p. 